\title{
Pricing Decisions of Two Complementary Products in a Fuzzy Environment
}

\author{
Li Wang, ${ }^{1}$ Jing Zhao, ${ }^{2}$ and Jie Wei ${ }^{3}$ \\ ${ }^{1}$ College of Aeronautical Automation, Civil Aviation University of China, Tianjin 300300, China \\ ${ }^{2}$ School of Science, Tianjin Polytechnic University, Tianjin 300160, China \\ ${ }^{3}$ General Courses Department, Military Transportation University, Tianjin 300161, China
}

Correspondence should be addressed to Jie Wei; sdtjwj@163.com

Received 26 November 2013; Accepted 12 February 2014; Published 16 March 2014

Academic Editor: Hao Shen

Copyright (C) $2014 \mathrm{Li}$ Wang et al. This is an open access article distributed under the Creative Commons Attribution License, which permits unrestricted use, distribution, and reproduction in any medium, provided the original work is properly cited.

\begin{abstract}
Pricing decisions of two complementary products in a fuzzy environment are considered in this paper. The purpose of this paper is to analyze the changes of the optimal retail pricing of two complementary products under two different decentralized decision scenarios (e.g., Nash game case and Stackelberg game case). As a reference model, the centralized pricing model is also established. The closed-form optimal pricing decisions of the two complementary products are obtained in the above three decision scenarios. Some interesting management insights into how pricing decisions vary with decision scenarios are given.
\end{abstract}

\section{Introduction}

Pricing plays an important role for a product because the long run survival of this product depends upon the firm's ability to increase its sales and obtain the maximum profit from the existing and new capital investment on this product. Furthermore, customers will inevitably apply price as a clue to form their attitude about a product if they lack complete information [1]. The price can be considered as one of the major factors for customers to purchase a product. Consequently, managers in the companies face the problem of establishing a pricing strategy that maximizes the profit over the sales horizon under various situations of price elasticity [2].

In the last several years, a considerable amount of research has studied the optimal pricing problem. Among these studies, some results are established by considering a single product or substitutable products. For example, Cai et al. [3] evaluated the impact of price discount contracts and pricing schemes on the dual-channel supply chain competition. Chiu et al. [4] used game theory to study some strategic actions for retailers to fight a price war. Tang and Yin [5] developed a base model with deterministic demand to examine how a retailer determines the order quantity and the retail price of two substitutable products jointly under the fixed and variable pricing strategies. Choi [6] studied the pricing decisions for fashion retailers with multiple information updating. Xia [7] studied competition between two coexisting suppliers in a two-echelon supply chain, where each supplier offers one type of the two substitutable products to multiple buyers.

On the other hand, some results are established by considering complementary products (products are complementary if a reduction in the price of one leads to an increase in demand for the other, which are the opposite of substitutable products). For example, Yue et al. [8] considered a market where customers need to buy two complementary goods as mixed bundle, offered by two separate firms. They presented a profit maximization model to obtain optimal strategies for a firm making decisions under information asymmetry. Mukhopadhyay et al. [9] showed that information sharing would benefit the leader firm but hurt the follower firm as well as the total system if the follower firm shares information unconditionally by considering a duopoly market where two separate firms offer complementary goods in a leaderfollower type move. The case of complementary products arises when customers have to buy more than one product at the same time to get the full utility of the goods [8]. Some complementary products are perfect complement because 
one product has to be consumed with another product, for example, a computer and an operating system, a left shoe and a right, pencils and erasers, DVD players and DVDs. Moreover, some complementary products are not perfect complement in that each product could be used separately, like a washer and a dryer. Pricing decisions for complementary products are not the same as it is for the more traditional products because one firm's pricing decision would affect the other firm's market performance and vice versa.

In fact, in order to make pricing schemes more effective, the uncertainties that happen in the real world cannot be neglected. Those uncertainties are usually associated with the product supply, the customer demand, and so on. There is an abundant literature that describes uncertainty in demand and/or lead time using probability distributions with known parameters, for example, Khan et al. [10], Zhang et al. [11], Guan and Zhao [12], and so forth. However, in many cases where there is little or no historical data available to the decision maker, perhaps due to recent changes in the supply chain, probability distribution may simply not be available or may not be easily or accurately estimated [13]. Moreover, other critical supply chain parameters, in particular the various costs that impact the system, are often ill-defined and may vary from time to time [14]. All of these situations raise challenges for using traditional supply chain models in practice [15].

In these situations, the uncertainty parameters can be approximately estimated by manager's judgements, intuitions, and experience and can be characterized as fuzzy variables [16]. Some researchers have already adopted fuzzy set theory to depict uncertainty in establishing the pricing model. For example, Zhao et al. [14] studied the pricing problem of substitutable products in a supply chain with one manufacturer and two competitive retailers. Zhou et al. [17] focused on the pricing problem of a single product with fuzzy customer demands. Sadjadi et al. [18] proposed a new possibilistic model which makes it easy to build the overall model based on experts' opinions. The proposed model is formulated in fuzzy geometric programming and the fuzzy decision is made using the recent advances in optimization techniques.

However, as far as we know, no research has considered the pricing problem of complementary products in a fuzzy supply chain environment. In this paper, we consider the pricing decisions of two complementary products in a fuzzy environment. The fuzziness is associated with the manufacturing costs and customer demands that are sensitive to the retail prices of two complementary products. The closedform optimal pricing decisions of the two complementary products under two different decentralized decision scenarios (e.g., Nash game case and Stackelberg game case) are established. As a reference model, the centralized pricing model is also considered and the corresponding closed-form optimal pricing decisions of the two complementary products are also given. Some interesting management insights into how pricing decisions vary with decision scenarios are given.

The rest of the paper is organized as follows. Section 2 gives the problem description and notations. Section 3 details our key analytical results. Numerical studies are given in Section 4. Some concluding comments are presented in Section 5.

\section{Problem Description}

Consider a supply chain of two complementary products with two manufacturers (labeled manufacturer 1 and manufacturer 2). The manufacturer $i$ produces a product $i$ with unit manufacturing cost $\widetilde{c}_{i}$ and sells it to the consumer with unit retail price $p_{i}, i=1,2$. We assume that products 1 and 2 are functionally complementary products with each other and there are three groups of consumers in the market. Group 1 buys product 1 only. Group 2 buys both products 1 and 2 , while group 3 buys product 2 only. We also assume that there is no price discrimination among the three consumer groups and all activities occur in a single period. The two manufacturers must make their pricing decisions in order to achieve their own maximal profits, respectively, and behave as if they have perfect information of the demands and the cost structures of other manufacturer.

Similar to Mukhopadhyay et al. [9], we assume that the demand of product 1 from group 1 is expressed as

$$
D_{11}\left(p_{1}\right)=\beta \widetilde{a}-\widetilde{\beta}_{1} p_{1}
$$

the demand of product 2 from group 3 is given as

$$
D_{23}\left(p_{2}\right)=\gamma \widetilde{a}-\widetilde{\beta}_{2} p_{2} ;
$$

the demand of products 1 and 2 from group 2 are defined as, respectively,

$$
\begin{aligned}
& D_{12}\left(p_{1}, p_{2}\right)=\widetilde{a}-\widetilde{\beta}_{11} p_{1}-\widetilde{\beta}_{12} p_{2}, \\
& D_{22}\left(p_{1}, p_{2}\right)=\widetilde{a}-\widetilde{\beta}_{22} p_{2}-\widetilde{\beta}_{21} p_{1},
\end{aligned}
$$

where the parameter $\tilde{a}$ denotes the primary demand of product 1 /product 2 from group 2, namely, when both retail prices $p_{1}$ and $p_{2}$ are zero, the demand for product 1 is the same as that for product 2 and is equal to $\tilde{a}, \beta \widetilde{a}$ denotes the primary demand of product 1 from group 1 , and $\gamma \tilde{a}$ denotes the primary demand of product 2 from group 3 , where $0<\beta<1$, $0<\gamma<1$. The parameters $\widetilde{\beta}_{1}, \widetilde{\beta}_{2}, \widetilde{\beta}_{11}, \widetilde{\beta}_{12}, \widetilde{\beta}_{21}$, and $\widetilde{\beta}_{22}$ are all price elasticities and the parameter $\widetilde{\beta}_{1}$ denotes the measure of the responsiveness of product 1's market demand from group 1 to its own retail price; the parameter $\widetilde{\beta}_{2}$ denotes the measure of the responsiveness of product 2's market demand from group 3 to its own retail price; the parameter $\widetilde{\beta}_{11}$ denotes the measure of the responsiveness of product 1's market demand from group 2 to its own retail price; the parameter $\widetilde{\beta}_{22}$ denotes the measure of the responsiveness of product 2's market demand from group 2 to its own retail price; the parameter $\widetilde{\beta}_{12}$ denotes the measure of the responsiveness of products 1's market demand from group 2 to products 2's retail price; and the parameter $\widetilde{\beta}_{21}$ denotes the measure of the responsiveness of products 2's market demand from group 2 to products 1's retail price. We assume that parameters $\widetilde{c}_{1}, \widetilde{c}_{2}, \widetilde{a}, \widetilde{\beta}_{1}, \widetilde{\beta}_{2}$, 
$\widetilde{\beta}_{11}, \widetilde{\beta}_{12}, \widetilde{\beta}_{21}$, and $\widetilde{\beta}_{22}$ are all independent nonnegative fuzzy variables and the expected values $E\left[\widetilde{\beta}_{11}\right], E\left[\widetilde{\beta}_{12}\right], E\left[\widetilde{\beta}_{22}\right]$, and $E\left[\widetilde{\beta}_{21}\right]$ satisfy $E\left[\widetilde{\beta}_{11}\right]>E\left[\widetilde{\beta}_{12}\right]>0$ and $E\left[\widetilde{\beta}_{22}\right]>E\left[\widetilde{\beta}_{21}\right]>0$, which means that the expected demand of product 1 (or 2) from group 2 is more sensitive to the changes in its own price than to the changes in the price of its complementary product 2 (or 1). This assumption is reasonable in reality.

The total demand for product 1 (denoted as $D_{1}\left(p_{1}, p_{2}\right)$ ) is the sum of its demands from three groups and is given as

$$
D_{1}\left(p_{1}, p_{2}\right)=(1+\beta) \widetilde{a}-\left(\widetilde{\beta}_{1}+\widetilde{\beta}_{11}\right) p_{1}-\widetilde{\beta}_{12} p_{2} .
$$

Similarly, the total demand for product 2 (denoted as $\left.D_{2}\left(p_{1}, p_{2}\right)\right)$ is the sum of its demands from three groups and is given as

$$
D_{2}\left(p_{1}, p_{2}\right)=(1+\gamma) \widetilde{a}-\left(\widetilde{\beta}_{2}+\widetilde{\beta}_{22}\right) p_{2}-\widetilde{\beta}_{21} p_{1} .
$$

The profits of manufacturer 1 (denoted as $\pi_{m 1}\left(p_{1}, p_{2}\right)$ ) and manufacturer 2 (denoted as $\pi_{m 2}\left(p_{1}, p_{2}\right)$ ) can be expressed as follows, respectively:

$$
\begin{aligned}
\pi_{m 1}\left(p_{1}, p_{2}\right) & =\left(p_{1}-\widetilde{c}_{1}\right) D_{1}\left(p_{1}, p_{2}\right) \\
& =\left(p_{1}-\widetilde{c}_{1}\right)\left((1+\beta) \widetilde{a}-\left(\widetilde{\beta}_{1}+\widetilde{\beta}_{11}\right) p_{1}-\widetilde{\beta}_{12} p_{2}\right), \\
\pi_{m 2}\left(p_{1}, p_{2}\right) & =\left(p_{2}-\widetilde{c}_{2}\right) D_{2}\left(p_{1}, p_{2}\right) \\
& =\left(p_{2}-\widetilde{c}_{2}\right)\left((1+\gamma) \tilde{a}-\left(\widetilde{\beta}_{2}+\widetilde{\beta}_{22}\right) p_{2}-\widetilde{\beta}_{21} p_{1}\right) .
\end{aligned}
$$

The total profit (denoted as $\pi_{c}\left(p_{1}, p_{2}\right)$ ) of manufacturer 1 and manufacturer 2 can be described as

$$
\begin{aligned}
\pi_{c}\left(p_{1}, p_{2}\right)= & \pi_{m 1}\left(p_{1}, p_{2}\right)+\pi_{m 2}\left(p_{1}, p_{2}\right) \\
= & \left(p_{1}-\widetilde{c}_{1}\right)\left((1+\beta) \widetilde{a}-\left(\widetilde{\beta}_{1}+\widetilde{\beta}_{11}\right) p_{1}-\widetilde{\beta}_{12} p_{2}\right) \\
& +\left(p_{2}-\widetilde{c}_{2}\right)\left((1+\gamma) \widetilde{a}-\left(\widetilde{\beta}_{2}+\widetilde{\beta}_{22}\right) p_{2}-\widetilde{\beta}_{21} p_{1}\right) .
\end{aligned}
$$

\section{Analytical Results}

3.1. Centralized Pricing Model (CD Model). As a benchmark to evaluate pricing decisions under different decision cases, we first examine the centralized pricing model (namely, assume that there is one entity who aims to optimize the total profit of manufacturer 1 and manufacturer 2). The two manufacturers' pricing decisions are fully coordinated in this case.

According to the above description, we know that the objective of the centralized pricing case is to maximize the expected total profit of manufacturer 1 and manufacturer 2 , which can be denoted as

$$
\begin{aligned}
& \max _{p_{1}, p_{2}} E\left[\pi_{c}\left(p_{1}, p_{2}\right)\right] \\
&=\max _{p_{1}, p_{2}} E[ {\left[\left(p_{1}-\widetilde{c}_{1}\right)\left((1+\beta) \widetilde{a}-\left(\widetilde{\beta}_{1}+\widetilde{\beta}_{11}\right) p_{1}-\widetilde{\beta}_{12} p_{2}\right)\right.} \\
&\left.+\left(p_{2}-\widetilde{c}_{2}\right)\left((1+\gamma) \widetilde{a}-\left(\widetilde{\beta}_{2}+\widetilde{\beta}_{22}\right) p_{2}-\widetilde{\beta}_{21} p_{1}\right)\right] .
\end{aligned}
$$

Proposition 1. In the centralized pricing case, the optimal retail prices (denoted as $p_{c 1}^{*}$ and $p_{c 2}^{*}$, resp.,) of the two complementary products are given as

$$
\begin{aligned}
& p_{c 1}^{*}=\frac{A_{2} A_{5}-A_{1} A_{4}}{A_{5}^{2}-A_{3} A_{4}}, \\
& p_{c 2}^{*}=\frac{A_{1} A_{5}-A_{2} A_{3}}{A_{5}^{2}-A_{3} A_{4}},
\end{aligned}
$$

where

$$
\begin{aligned}
& A_{1}=(1+\beta) E[\widetilde{a}]+E\left[\widetilde{c}_{1} \widetilde{\beta}_{1}\right]+E\left[\widetilde{c}_{1} \widetilde{\beta}_{11}\right]+E\left[\widetilde{c}_{2} \widetilde{\beta}_{21}\right], \\
& A_{2}=(1+\gamma) E[\widetilde{a}]+E\left[\widetilde{c}_{2} \widetilde{\beta}_{2}\right]+E\left[\widetilde{c}_{2} \widetilde{\beta}_{22}\right]+E\left[\widetilde{c}_{1} \widetilde{\beta}_{12}\right], \\
& A_{3}=2\left(E\left[\widetilde{\beta}_{1}\right]+E\left[\widetilde{\beta}_{11}\right]\right), \quad A_{4}=2\left(E\left[\widetilde{\beta}_{2}\right]+E\left[\widetilde{\beta}_{22}\right]\right), \\
& A_{5}=\left(E\left[\widetilde{\beta}_{12}\right]+E\left[\widetilde{\beta}_{21}\right]\right) .
\end{aligned}
$$

Proof. Using (7), the expected total profit $E\left[\pi_{c}\left(p_{1}, p_{2}\right)\right]$ of manufacturer 1 and manufacturer 2 can be given as

$$
\begin{aligned}
E\left[\pi_{c}\left(p_{1}, p_{2}\right)\right]= & (1+\beta) E[\widetilde{a}] p_{1}-\left(E\left[\widetilde{\beta}_{1}\right]+E\left[\widetilde{\beta}_{11}\right]\right) p_{1}^{2} \\
& -E\left[\widetilde{\beta}_{12}\right] p_{1} p_{2}+E\left[\widetilde{c}_{1} \widetilde{\beta}_{12}\right] p_{2} \\
& -\frac{1+\beta}{2} \int_{0}^{1}\left(\widetilde{c}_{1 \alpha}^{U} \widetilde{a}_{\alpha}^{L}+\widetilde{c}_{1 \alpha}^{L} \widetilde{a}_{\alpha}^{U}\right) d \alpha \\
& +\left(E\left[\widetilde{c}_{1} \widetilde{\beta}_{1}\right]+E\left[\widetilde{c}_{1} \widetilde{\beta}_{11}\right]\right) p_{1} \\
& +(1+\gamma) E[\widetilde{a}] p_{2} \\
& -\frac{1+\gamma}{2} \int_{0}^{1}\left(\widetilde{c}_{2 \alpha}^{U} \widetilde{a}_{\alpha}^{L}+\widetilde{c}_{2 \alpha}^{L} \widetilde{a}_{\alpha}^{U}\right) d \alpha \\
& -\left(E\left[\widetilde{\beta}_{2}\right]+E\left[\widetilde{\beta}_{22}\right]\right) p_{2}^{2}+E\left[\widetilde{c}_{2} \widetilde{\beta}_{21}\right] p_{1} \\
& -E\left[\widetilde{\beta}_{21}\right] p_{1} p_{2}+\left(E\left[\widetilde{c}_{2} \widetilde{\beta}_{2}\right]+E\left[\widetilde{c}_{2} \widetilde{\beta}_{22}\right]\right) p_{2} .
\end{aligned}
$$

The first and second order partial derivatives of $\pi_{c}\left(p_{1}, p_{2}\right)$ to $p_{1}$ and $p_{2}$ can be shown as

$$
\begin{aligned}
\frac{\partial E\left[\pi_{c}\left(p_{1}, p_{2}\right)\right]}{\partial p_{1}}= & -2\left(E\left[\widetilde{\beta}_{1}\right]+E\left[\widetilde{\beta}_{11}\right]\right) p_{1} \\
& -\left(E\left[\widetilde{\beta}_{12}\right]+E\left[\widetilde{\beta}_{21}\right]\right) p_{2}+(1+\beta) E[\widetilde{a}] \\
& +E\left[\widetilde{c}_{1} \widetilde{\beta}_{1}\right]+E\left[\widetilde{c}_{1} \widetilde{\beta}_{11}\right]+E\left[\widetilde{c}_{2} \widetilde{\beta}_{21}\right]
\end{aligned}
$$




$$
\begin{aligned}
\frac{\partial E\left[\pi_{c}\left(p_{1}, p_{2}\right)\right]}{\partial p_{2}}= & -2\left(E\left[\widetilde{\beta}_{2}\right]+E\left[\widetilde{\beta}_{22}\right]\right) p_{2} \\
& -\left(E\left[\widetilde{\beta}_{12}\right]+E\left[\widetilde{\beta}_{21}\right]\right) p_{1}+(1+\gamma) E[\widetilde{a}] \\
& +E\left[\widetilde{c}_{2} \widetilde{\beta}_{2}\right]+E\left[\widetilde{c}_{2} \widetilde{\beta}_{22}\right]+E\left[\widetilde{c}_{1} \widetilde{\beta}_{12}\right],
\end{aligned}
$$

$$
\begin{aligned}
\frac{\partial^{2} E\left[\pi_{c}\left(p_{1}, p_{2}\right)\right]}{\partial p_{1}^{2}}= & -2\left(E\left[\widetilde{\beta}_{1}\right]+E\left[\widetilde{\beta}_{11}\right]\right), \\
\frac{\partial^{2} E\left[\pi_{c}\left(p_{1}, p_{2}\right)\right]}{\partial p_{2}^{2}}= & -2\left(E\left[\widetilde{\beta}_{2}\right]+E\left[\widetilde{\beta}_{22}\right]\right), \\
\frac{\partial^{2} E\left[\pi_{c}\left(p_{1}, p_{2}\right)\right]}{\partial p_{1} \partial p_{2}} & =\frac{\partial^{2} E\left[\pi_{c}\left(p_{1}, p_{2}\right)\right]}{\partial p_{2} \partial p_{1}} \\
& =-\left(E\left[\widetilde{\beta}_{12}\right]+E\left[\widetilde{\beta}_{21}\right]\right) .
\end{aligned}
$$

With (13), we can see that the expected profit $E\left[\pi_{c}\left(p_{1}\right.\right.$, $\left.\left.p_{2}\right)\right]$ is jointly concave in $p_{1}$ and $p_{2}$. Equation (9) can be easily obtained by setting (12) to zero and solving them simultaneously, so Proposition 1 is proven.

3.2. Decentralized Pricing Models. In this section, we assume that the two manufacturers are independent with each other in the supply chain system. They make their own decision with the objective to maximize their own expected profit. The game-theoretical approach is used to analyze the pricing decision models established in the following. The assumption regarding which firm possesses the bargaining power in the supply chain system can influence how the pricing models are solved.

3.2.1. Pricing Model in Nash Game (NG Model). The NG model represents a market in which there are relatively small to medium-sized manufacturers. The two manufacturers have the identical market power; they determine their strategies independently and simultaneously. Because manufacturer 1 cannot dominate the market over manufacturer 2, his price decision is conditioned on the retail price of product 2. On the contrary, manufacturer 2 must make his own retail price, simultaneously, conditional on the product l's retail price. This situation is called a Nash game and the solution to this structure is the Nash equilibrium. Therefore, we have the following results expressed in Proposition 2.

Proposition 2. In Nash game case, the two manufacturers' optimal retail prices (denoted as $p_{n 1}^{*}$ and $p_{n 2}^{*}$, resp.,) are

$$
\begin{aligned}
& p_{n 1}^{*}=\frac{E\left[\widetilde{\beta}_{12}\right] B_{3}-B_{1} B_{4}}{E\left[\widetilde{\beta}_{12}\right] E\left[\widetilde{\beta}_{21}\right]-B_{2} B_{4}}, \\
& p_{n 2}^{*}=\frac{E\left[\widetilde{\beta}_{21}\right] B_{1}-B_{2} B_{3}}{E\left[\widetilde{\beta}_{12}\right] E\left[\widetilde{\beta}_{21}\right]-B_{2} B_{4}},
\end{aligned}
$$

where

$$
\begin{aligned}
& B_{1}=(1+\beta) E[\widetilde{a}]+E\left[\widetilde{c}_{1} \widetilde{\beta}_{1}\right]+E\left[\widetilde{c}_{1} \widetilde{\beta}_{11}\right], \\
& B_{2}=2\left(E\left[\widetilde{\beta}_{1}\right]+E\left[\widetilde{\beta}_{11}\right]\right), \\
& B_{3}=(1+\gamma) E[\widetilde{a}]+E\left[\widetilde{c}_{2} \widetilde{\beta}_{2}\right]+E\left[\widetilde{c}_{2} \widetilde{\beta}_{22}\right], \\
& B_{4}=2\left(E\left[\widetilde{\beta}_{2}\right]+E\left[\widetilde{\beta}_{22}\right]\right) .
\end{aligned}
$$

Proof. Using (6), the expected total profits $E\left[\pi_{m 1}\left(p_{1}, p_{2}\right)\right]$ of manufacturer 1 and $E\left[\pi_{m 2}\left(p_{1}, p_{2}\right)\right]$ of manufacturer 2 can be given as, respectively,

$$
\begin{aligned}
E\left[\pi_{m 1}\left(p_{1}, p_{2}\right)\right]= & (1+\beta) E[\widetilde{a}] p_{1}-\left(E\left[\widetilde{\beta}_{1}\right]+E\left[\widetilde{\beta}_{11}\right]\right) p_{1}^{2} \\
& -E\left[\widetilde{\beta}_{12}\right] p_{1} p_{2}+E\left[\widetilde{c}_{1} \widetilde{\beta}_{12}\right] p_{2} \\
& -\frac{1+\beta}{2} \int_{0}^{1}\left(\widetilde{c}_{1 \alpha}^{U} \widetilde{a}_{\alpha}^{L}+\widetilde{c}_{1 \alpha}^{L} \widetilde{a}_{\alpha}^{U}\right) d \alpha \\
& +\left(E\left[\widetilde{c}_{1} \widetilde{\beta}_{1}\right]+E\left[\widetilde{c}_{1} \widetilde{\beta}_{11}\right]\right) p_{1}, \\
E\left[\pi_{m 2}\left(p_{1}, p_{2}\right)\right]= & (1+\gamma) E[\widetilde{a}] p_{2}-\left(E\left[\widetilde{\beta}_{2}\right]+E\left[\widetilde{\beta}_{22}\right]\right) p_{2}^{2} \\
& -E\left[\widetilde{\beta}_{21}\right] p_{1} p_{2}+E\left[\widetilde{c}_{2} \widetilde{\beta}_{21}\right] p_{1} \\
& -\frac{1+\gamma}{2} \int_{0}^{1}\left(\widetilde{c}_{2 \alpha}^{U} \widetilde{a}_{\alpha}^{L}+\widetilde{c}_{2 \alpha}^{L} \widetilde{a}_{\alpha}^{U}\right) d \alpha \\
& +\left(E\left[\widetilde{c}_{2} \widetilde{\beta}_{2}\right]+E\left[\widetilde{c}_{2} \widetilde{\beta}_{22}\right]\right) p_{2} .
\end{aligned}
$$

The first and second order derivatives of $E\left[\pi_{m 1}\left(p_{1}, p_{2}\right)\right]$ to $p_{1}$ and $E\left[\pi_{m 2}\left(p_{1}, p_{2}\right)\right]$ to $p_{2}$ can be shown as

$$
\begin{aligned}
\frac{\partial E\left[\pi_{m 1}\left(p_{1}, p_{2}\right)\right]}{\partial p_{1}}= & -2\left(E\left[\widetilde{\beta}_{1}\right]+E\left[\widetilde{\beta}_{11}\right]\right) p_{1}-E\left[\widetilde{\beta}_{12}\right] p_{2} \\
& +(1+\beta) E[\widetilde{a}]+E\left[\widetilde{c}_{1} \widetilde{\beta}_{1}\right]+E\left[\widetilde{c}_{1} \widetilde{\beta}_{11}\right], \\
\frac{\partial E\left[\pi_{m 2}\left(p_{1}, p_{2}\right)\right]}{\partial p_{2}}= & -2\left(E\left[\widetilde{\beta}_{2}\right]+E\left[\widetilde{\beta}_{22}\right]\right) p_{2}-E\left[\widetilde{\beta}_{21}\right] p_{1} \\
& +(1+\gamma) E[\widetilde{a}]+E\left[\widetilde{c}_{2} \widetilde{\beta}_{2}\right]+E\left[\widetilde{c}_{2} \widetilde{\beta}_{22}\right],
\end{aligned}
$$

$\frac{\partial^{2} E\left[\pi_{m 1}\left(p_{1}, p_{2}\right)\right]}{\partial p_{1}^{2}}=-2\left(E\left[\widetilde{\beta}_{1}\right]+E\left[\widetilde{\beta}_{11}\right]\right)<0$,

$\frac{\partial^{2} E\left[\pi_{m 2}\left(p_{1}, p_{2}\right)\right]}{\partial p_{2}^{2}}=-2\left(E\left[\widetilde{\beta}_{2}\right]+E\left[\widetilde{\beta}_{12}\right]\right)<0$.

With (20) and (21), we know that the expected profits $E\left[\pi_{m 1}\left(p_{1}, p_{2}\right)\right]$ is concave in $p_{1}$ and $E\left[\pi_{m 2}\left(p_{1}, p_{2}\right)\right]$ is concave in $p_{2}$.

By setting (18) and (19) equal to zero and solving for $p_{1}$ and $p_{2}$ simultaneously, the optimal retail prices $p_{n 1}^{*}$ and 
$p_{n 2}^{*}$ expressed as in (14) can be obtained, so Proposition 2 is proven.

3.2.2. Pricing Model in Stackelberg Game (SG Model). The SG model arises in a market where one manufacturer's size is smaller compared to the other manufacturers. So, the two manufacturers have different market powers, they determine their strategies independently and sequentially. Variation in the bargaining power can create one of the following two scenarios besides the above Nash game: (1) manufacturer 1 lead Stackelberg game, manufacturer 1 holds more bargaining power than manufacturer 2 and thus is the Stackelberg leader and (2) Manufacturer 2 lead Stackelberg game, manufacturer 2 holds more bargaining power than manufacturer 1 and thus is the Stackelberg leader. Without loss of generality, in this section, we assume that manufacturer 1 acts as the Stackelberg leader and manufacturer 2 acts as the Stackelberg follower.

When manufacturer 1 acts as the Stackelberg leader and manufacturer 2 acts as the Stackelberg follower. Manufacturer 1 first announces the retail price of the product 1 . Having the information about the decision of manufacturer 1 , manufacturer 2 would then use it to maximize his expected profit. So, we have the following result given as in Proposition 3.

Proposition 3. When the two manufacturers play Stackelberg game and manufacturer 1 is the game leader, manufacturer 2's optimal retail price (denoted as $p_{s 2}\left(p_{1}\right)$ ), given earlier decision made by manufacturer 1 is $p_{1}$, is

$$
p_{s 2}\left(p_{1}\right)=F_{1}-F_{2} p_{1}
$$

where

$$
\begin{aligned}
F_{1} & =\frac{(1+\gamma) E[\widetilde{a}]+E\left[\widetilde{c}_{2} \widetilde{\beta}_{2}\right]+E\left[\widetilde{c}_{2} \widetilde{\beta}_{22}\right]}{2\left(E\left[\widetilde{\beta}_{2}\right]+E\left[\widetilde{\beta}_{22}\right]\right)}, \\
F_{2} & =\frac{E\left[\widetilde{\beta}_{21}\right]}{2\left(E\left[\widetilde{\beta}_{2}\right]+E\left[\widetilde{\beta}_{22}\right]\right)} .
\end{aligned}
$$

Proof. Using (21), we know that the expected profit $E\left[\pi_{m 1}\left(p_{1}, p_{2}\right)\right]$ is concave in $p_{1}$ for given $p_{2}$. Setting (19) to zero and solving it, we can easily have (22), so Proposition 3 is proven.

Proposition 4. When the two manufacturers play Stackelberg game and manufacturer 1 is the game leader, manufacturer 1's optimal retail price (denoted as $p_{s 1}^{*}$ ) is given as

$$
p_{s 1}^{*}=\frac{F_{3}}{F_{4}},
$$

where $F_{3}=(1+\beta) E[\widetilde{a}]+E\left[\widetilde{c}_{1} \widetilde{\beta}_{1}\right]+E\left[\widetilde{c}_{1} \widetilde{\beta}_{11}\right]-E\left[\widetilde{c}_{1} \widetilde{\beta}_{12}\right] F_{2}-$ $E\left[\widetilde{\beta}_{12}\right] F_{1}, F_{4}=2 E\left[\widetilde{\beta}_{1}\right]+2 E\left[\widetilde{\beta}_{11}\right]+2 E\left[\widetilde{\beta}_{12}\right] F_{2}$, and $F_{1}, F_{2}$ are given as in Proposition 3.
Proof. It follows from (16) and (22) that the first order and second order derivatives of $E\left[\pi_{m 1}\left(p_{1}, p_{2}\right)\right]$ to $p_{1}$ can be shown as

$$
\begin{aligned}
\frac{\partial E\left[\pi_{m 1}\left(p_{1}, p_{2}\right)\right]}{\partial p_{1}} \\
=-\left(2 E\left[\widetilde{\beta}_{1}\right]+2 E\left[\widetilde{\beta}_{11}\right]-2 E\left[\widetilde{\beta}_{12}\right] F_{2}\right) p_{1} \\
+(1+\beta) E[\widetilde{a}]+E\left[\widetilde{c}_{1} \widetilde{\beta}_{1}\right] \\
+E\left[\widetilde{c}_{1} \widetilde{\beta}_{11}\right]-E\left[\widetilde{c}_{1} \widetilde{\beta}_{12}\right] F_{2}-E\left[\widetilde{\beta}_{12}\right] F_{1}, \\
\\
\quad \frac{\partial^{2} E\left[\pi_{m 1}\left(p_{1}, p_{2}\right)\right]}{\partial p_{1}^{2}}=-F_{4}<0 .
\end{aligned}
$$

Using (26), we know that $E\left[\pi_{m 1}\left(p_{1}, p_{2}\right)\right]$ is concave in $p_{1}$. Setting (25) to zero and solving it, we can easily have (24), so Proposition 4 is proven.

Proposition 5. When the two manufacturers play Stackelberg game and manufacturer 1 is the game leader, manufacturer 2's optimal retail price (denoted as $p_{s 2}^{*}$ ) is

$$
p_{s 2}^{*}=F_{1}-\frac{F_{2} F_{3}}{F_{4}}
$$

where $F_{1}, F_{2}, F_{3}$, and $F_{4}$ are defined as in Propositions 3 and 4.

Proof. By Propositions 3 and 4, we can easily see that Proposition 5 holds.

\section{Numerical Studies}

Because the optimal pricing strategy obtained in this paper is in a very complicated form, especially the $\alpha$-optimistic value and $\alpha$-pessimistic value of fuzzy variable contained in the results, we have to use numerical examples to compare the results obtained from the above three different decision scenarios and to study the behavior of firms facing changing environment. Although in the following only a particular set of data is reported, we actually have conducted some computational experiments from which similar observations have been obtained.

In this section, we compare the analytical results obtained from the above three different decision scenarios using numerical approach and study the behavior of the two firms facing changing decision environment. Using the analytical results obtained from the above three different decision scenarios, we can easily see that the expressions of the optimal retail prices and maximum expected profits under different decision scenarios.

Here, we consider two manufacturers of a consumer electronics manufacturing industry in China, the data used in the following numerical example are from them, which satisfies or closely satisfies the assumptions of this paper. These data have been appropriately manipulated, for example, standardization and non-dimensionalization, before being employed, which satisfies or closely complies with certain 
assumptions of this research. We think these data can represent the real-world condition as closely as possible due to the difficulty of accessing the actual industry data.

The relationship between linguistic expressions and triangular fuzzy variables for manufacturing cost, primary demand, and price elasticity are often determined by experts' experiences, as shown in Table 1.

Consider the case where the manufacturing costs $\widetilde{c}_{1}$ and $\widetilde{c}_{2}$ are high $\left(\widetilde{c}_{1}\right.$ is about $8, \widetilde{c}_{2}$ is about 16$)$, the primary demand $\tilde{a}$ is large $(\tilde{a}$ is about 850$)$, price elasticities $\widetilde{\beta}_{1}, \widetilde{\beta}_{2}, \widetilde{\beta}_{11}, \widetilde{\beta}_{12}$, $\widetilde{\beta}_{21}$, and $\widetilde{\beta}_{22}$ are all sensitive $\left(\widetilde{\beta}_{1}\right.$ is about $6, \widetilde{\beta}_{2}$ is about $7, \widetilde{\beta}_{11}$ is about $9, \widetilde{\beta}_{12}$ is about $7, \widetilde{\beta}_{21}$ is about 6 , and $\widetilde{\beta}_{22}$ is about 8 ), and $\beta=0.6, \gamma=0.7$. Using Table $1, \widetilde{c}_{1}=(6,8,9), \widetilde{c}_{2}=(14,16,19)$, $\tilde{a}=(650,850,950), \widetilde{\beta}_{1}=(3,6,8), \widetilde{\beta}_{2}=(5,7,9), \widetilde{\beta}_{11}=$ $(6,9,11), \widetilde{\beta}_{12}=(5,7,8), \widetilde{\beta}_{21}=(4,6,8)$, and $\widetilde{\beta}_{22}=(7,8,9)$ are all triangular fuzzy variables. By using Definition 7 [19], the expected values are

$$
\begin{aligned}
& E\left[\widetilde{c}_{1}\right]=\frac{6+2 \times 8+9}{4}=7.75, \\
& E\left[\widetilde{c}_{2}\right]=\frac{14+2 \times 16+19}{4}=16.25, \\
& E[\widetilde{a}]=\frac{650+2 \times 850+950}{4}=825, \\
& E\left[\widetilde{\beta}_{1}\right]=\frac{3+2 \times 6+8}{4}=5.75, \\
& E\left[\widetilde{\beta}_{2}\right]=\frac{5+2 \times 7+9}{4}=7, \\
& E\left[\widetilde{\beta}_{11}\right]=\frac{6+2 \times 9+11}{4}=8.75, \\
& E\left[\widetilde{\beta}_{12}\right]=\frac{5+2 \times 7+9}{4}=7, \\
& E\left[\widetilde{\beta}_{21}\right]=\frac{4+2 \times 6+8}{4}=6, \\
& E\left[\widetilde{\beta}_{22}\right]=\frac{7+2 \times 8+9}{4}=8 .
\end{aligned}
$$

It follows from Definition 5 [19] that the $\alpha$-optimistic value and $\alpha$-pessimistic value of $\widetilde{c}_{1}, \widetilde{c}_{2}, \tilde{a}, \widetilde{\beta}_{1}, \widetilde{\beta}_{2}, \widetilde{\beta}_{11}, \widetilde{\beta}_{12}, \widetilde{\beta}_{21}$, and $\widetilde{\beta}_{22}$ are, respectively,

$$
\begin{array}{ccc}
\widetilde{c}_{1 \alpha}^{L}=6+2 \alpha, & \widetilde{c}_{2 \alpha}^{L}=14+2 \alpha, & \widetilde{a}_{\alpha}^{L}=650+200 \alpha, \\
\widetilde{\beta}_{1 \alpha}^{L}=6+3 \alpha, & \widetilde{\beta}_{2 \alpha}^{L}=5+2 \alpha, & \widetilde{\beta}_{11 \alpha}^{L}=6+3 \alpha, \\
\widetilde{\beta}_{12 \alpha}^{L}=5+2 \alpha, & \widetilde{\beta}_{21 \alpha}^{L}=4+2 \alpha, & \widetilde{\beta}_{22 \alpha}^{L}=7+\alpha, \\
\widetilde{c}_{1 \alpha}^{U}=9-\alpha, & \widetilde{c}_{2 \alpha}^{U}=19-3 \alpha, & \widetilde{a}_{\alpha}^{U}=950-100 \alpha, \\
\widetilde{\beta}_{1 \alpha}^{U}=8-2 \alpha, & \widetilde{\beta}_{2 \alpha}^{U}=9-2 \alpha, & \widetilde{\beta}_{11 \alpha}^{U}=11-2 \alpha, \\
\widetilde{\beta}_{12 \alpha}^{U}=9-2 \alpha, & \widetilde{\beta}_{21 \alpha}^{U}=8-2 \alpha, & \widetilde{\beta}_{22 \alpha}^{U}=9-\alpha .
\end{array}
$$

TABLE 1: Relation between linguistic expression and triangular fuzzy variable.

\begin{tabular}{lcc}
\hline & Linguistic expression & $\begin{array}{c}\text { Triangular fuzzy } \\
\text { variable }\end{array}$ \\
\hline \multirow{2}{*}{ Manufacturing cost $\widetilde{c}_{1}$} & High (about 8) & $(6,8,9)$ \\
& Medium (about 5) & $(4,5,6)$ \\
& Low (about 2) & $(1,2,4)$ \\
\hline \multirow{2}{*}{ Manufacturing cost $\widetilde{c}_{2}$} & High (about 16) & $(14,16,19)$ \\
& Medium (about 9) & $(7,9,13)$ \\
& Low (about 4) & $(2,4,6)$ \\
\hline \multirow{2}{*}{ Primary demand $\widetilde{a}$} & Large (about 850) & $(650,850,950)$ \\
& Small (about 300) & $(200,300,350)$ \\
\hline \multirow{2}{*}{ Price elasticity $\widetilde{\beta}_{1}$} & Very sensitive (about 11) & $(9,11,13)$ \\
& Sensitive (about 6) & $(3,6,8)$ \\
\hline \multirow{2}{*}{ Price elasticity $\widetilde{\beta}_{2}$} & Very sensitive (about 10) & $(8,10,11)$ \\
& Sensitive (about 7) & $(5,7,9)$ \\
\hline \multirow{2}{*}{ Price elasticity $\widetilde{\beta}_{11}$} & Very sensitive (about 14) & $(12,14,16)$ \\
& Sensitive (about 9) & $(6,9,11)$ \\
\hline \multirow{2}{*}{ Price elasticity $\widetilde{\beta}_{12}$} & Very sensitive (about 10) & $(8,10,11)$ \\
& Sensitive (about 7) & $(5,7,8)$ \\
\hline \multirow{2}{*}{ Price elasticity $\widetilde{\beta}_{21}$} & Very sensitive (about 9) & $(7,9,12)$ \\
\hline \multirow{2}{*}{ Price elasticity $\widetilde{\beta}_{22}$} & Sensitive (about 6) & $(4,6,8)$ \\
\hline & Very sensitive (about 11) & $(8,11,14)$ \\
& Sensitive (about 8) & $(7,8,9)$ \\
\hline
\end{tabular}

Using Lemmas 3 and 4 [19], we have $E\left[\widetilde{c}_{1} \widetilde{\beta}_{1}\right]=56.3333$, $E\left[\widetilde{c}_{1} \widetilde{\beta}_{11}\right]=69.0833, E\left[\widetilde{c}_{1} \widetilde{\beta}_{12}\right]=55.2500, E\left[\widetilde{c}_{1} \widetilde{\beta}_{21}\right]=47.5000$, $E\left[\widetilde{c}_{1} \widetilde{\beta}_{22}\right]=62.5000, E\left[\widetilde{c}_{2} \widetilde{\beta}_{2}\right]=115.4167, E\left[\widetilde{c}_{2} \widetilde{\beta}_{11}\right]=$ $144.2500, E\left[\widetilde{c}_{2} \widetilde{\beta}_{12}\right]=115.4167, E\left[\widetilde{c}_{2} \widetilde{\beta}_{21}\right]=99.1667$, $E\left[\widetilde{c}_{2} \widetilde{\beta}_{22}\right]=130.8333,(1 / 2) \int_{0}^{1}\left(\widetilde{c}_{1 \alpha}^{U} \widetilde{a}_{\alpha}^{L}+\widetilde{c}_{1 \alpha}^{L} \widetilde{a}_{\alpha}^{U}\right) d \alpha=6320.8$, and $(1 / 2) \int_{0}^{1}\left(\widetilde{c}_{2 \alpha}^{U} \widetilde{a}_{\alpha}^{L}+\widetilde{c}_{2 \alpha}^{L} \widetilde{a}_{\alpha}^{U}\right) d \alpha=13279$. Using the analytical results obtained in this paper, the corresponding numerical results are tabulated in Tables 2 and 3, respectively.

Discussions. From Tables 2 and 3, we can have the following results.

(1) The expected profit of the whole supply chain in CD model is the biggest one among the three pricing models, and the expected profit of the whole supply chain in NG model is the bigger one between the two decentralized pricing models. Moreover, both manufacturer 1 and manufacturer 2 achieve their own higher expected profits in NG model followed by SG model. This indicates that the NG game involving two manufacturers is more beneficial to the whole supply chain than their Stackelberg game; similarly, the NG game involving two manufacturers is more beneficial to each manufacturer than their Stackelberg game. From which one can see that the leader does not have the advantage to achieve higher expected profit in SG model, the whole supply chain and each manufacturer are better off when no channel member in a dominant position. This means that the leadership between the two manufacturers reduces profits of the whole supply chain and each manufacturer. 
TABLE 2: The optimal expected profits under different decision cases.

\begin{tabular}{lccc}
\hline Scenario & $E\left[\pi_{c}\right]$ & $E\left[\pi_{m 1}\right]$ & $E\left[\pi_{m 2}\right]$ \\
\hline CD model & 370580 & & \\
NG model & 26494 & 13915 & 12580 \\
SG model & 25292 & 13297 & 11995 \\
\hline
\end{tabular}

TABle 3: Optimal decisions of retail prices under different decision cases.

\begin{tabular}{lcc}
\hline Scenario & $p_{1}^{*}$ & $p_{2}^{*}$ \\
\hline CD model & 116.2764 & 41.8494 \\
NG model & 38.4315 & 47.2720 \\
SG model & 33.0081 & 48.3567 \\
\hline
\end{tabular}

(2) Table 3 tells us that the complementary product 1 achieve the highest retail price in CD model followed by NG model and achieves the lowest retail price in SG model. This seems counter-intuitive and needs our further exploration in the future. On the other hand, the complementary product 2 achieve the highest retail price in SG model followed by NG model and achieves the lowest retail price in CD model, which is consistent with our intuitions.

\section{Some Concluding Comments}

We analyze the pricing decisions for two complementary products under three different decision cases. As a benchmark to evaluate channel decision in different decision cases, we first develop the pricing model in centralized decision case and derive the optimal retail prices. We then establish the pricing models in two decentralized decision cases (e.g., NG model and SG model) and give the optimal retail prices. Finally, we provide comparison of the expected profits and optimal pricing decisions of the whole supply chain and each supply chain members. Some interesting insights into the economic behavior of firms are established in this paper.

However, our results are based upon some assumptions about the pricing models of complementary products. Thus, several extensions to the analysis in this paper are possible. First, this paper considers a case with linear-demand function in a single-period, one can study the case with other forms of demand function in multiple periods. Second, we assumed that both the two manufacturers have symmetric information about costs and demands. So, an extension would be to consider the supply chain with information asymmetry, such as asymmetry in cost information and demand information. Third, we can also consider the coordination of the supply chain under linear or isoelastic demand with symmetric and asymmetric information.

\section{Appendix}

\section{Preliminary on Fuzzy Set}

For the preliminary on fuzzy set used in this paper, see Appendix 1 in [14].

\section{Conflict of Interests}

The authors declare that there is no conflict of interests regarding the publication of this paper.

\section{Acknowledgments}

The authors gratefully acknowledge the support of the (i) National Natural Science Foundation of China (NSFC), Research Fund nos. 71001106, 71371186, and 91024002 for J. Wei; (ii) jointly funded projects of National Natural Science Foundation Committee and Civil Aviation Administration of China no. U1333111 and Fundamental Research Project of Central Universities no. ZXB 2011A003, for L. Wang; and (iii) NSFC Research Fund no. 71301116, for J. Zhao.

\section{References}

[1] R. Suri and K. B. Monroe, "The effects of time constraints on consumers' judgments of prices and products," Journal of Consumer Research, vol. 30, no. 1, pp. 92-104, 2003.

[2] S. Y. Sohn, T. H. Moon, and K. J. Seok, "Optimal pricing for mobile manufacturers in competitive market using genetic algorithm," Expert Systems with Applications, vol. 36, no. 2, pp. 3448-3453, 2009.

[3] G. Cai, Z. G. Zhang, and M. Zhang, "Game theoretical perspectives on dual-channel supply chain competition with price discounts and pricing schemes," International Journal of Production Economics, vol. 117, no. 1, pp. 80-96, 2009.

[4] C.-H. Chiu, T.-M. Choi, and D. Li, "Price wall or war: the pricing strategies for retailers," IEEE Transactions on Systems, Man, and Cybernetics A, vol. 39, no. 2, pp. 331-343, 2009.

[5] C. S. Tang and R. Yin, "Joint ordering and pricing strategies for managing substitutable products," Production and Operations Management, vol. 16, no. 1, pp. 138-153, 2007.

[6] T.-M. Choi, "Pre-season stocking and pricing decisions for fashion retailers with multiple information updating," International Journal of Production Economics, vol. 106, no. 1, pp. 146-170, 2007.

[7] Y. Xia, "Competitive strategies and market segmentation for suppliers with substitutable products," European Journal of Operational Research, vol. 210, no. 2, pp. 194-203, 2011.

[8] X. Yue, S. K. Mukhopadhyay, and X. Zhu, "A Bertrand model of pricing of complementary goods under information asymmetry," Journal of Business Research, vol. 59, no. 10-11, pp. 1182-1192, 2006.

[9] S. K. Mukhopadhyay, X. Yue, and X. Zhu, "A Stackelberg model of pricing of complementary goods under information asymmetry," International Journal of Production Economics, vol. 134, no. 2, pp. 424-433, 2011.

[10] M. Khan, M. Y. Jaber, and M. Bonney, "An economic order quantity (EOQ) for items with imperfect quality and inspection errors," International Journal of Production Economics, vol. 133, no. 1, pp. 113-118, 2011.

[11] J.-L. Zhang, J. Chen, and C.-Y. Lee, "Joint optimization on pricing, promotion and inventory control with stochastic demand," International Journal of Production Economics, vol. 116, no. 2, pp. 190-198, 2008.

[12] R. Guan and X. Zhao, "Pricing and inventory management in a system with multiple competing retailers under (r, Q) policies," 
Computers \& Operations Research, vol. 38, no. 9, pp. 1294-1304, 2011.

[13] Y. Xie, D. Petrovic, and K. Burnham, "A heuristic procedure for the two-level control of serial supply chains under fuzzy customer demand," International Journal of Production Economics, vol. 102, no. 1, pp. 37-50, 2006.

[14] J. Zhao, W. Tang, and J. Wei, "Pricing decision for substitutable products with retail competition in a fuzzy environment," International Journal of Production Economics, vol. 135, no. 1, pp. 144-153, 2012.

[15] R. Handfield, D. Warsing, and X. Wu, “(Q, r) inventory policies in a fuzzy uncertain supply chain environment," European Journal of Operational Research, vol. 197, no. 2, pp. 609-619, 2009.

[16] H.-J. Zimmermann, "An application-oriented view of modeling uncertainty," European Journal of Operational Research, vol. 122, no. 2, pp. 190-198, 2000.

[17] C. Zhou, R. Zhao, and W. Tang, "Two-echlon supply chain games in a fuzzy environment," Computers \& Industrial Engineering, vol. 55, no. 2, pp. 390-405, 2008.

[18] S. J. Sadjadi, M. Ghazanfari, and A. Yousefli, "Fuzzy pricing and marketing planning model: a possibilistic geometric programming approach," Expert Systems with Applications, vol. 37, no. 4, pp. 3392-3397, 2010.

[19] J. Wei and J. Zhao, "Pricing decisions with retail competition in a fuzzy closed-loop supply chain," Expert Systems with Applications, vol. 38, no. 9, pp. 11209-11216, 2011. 


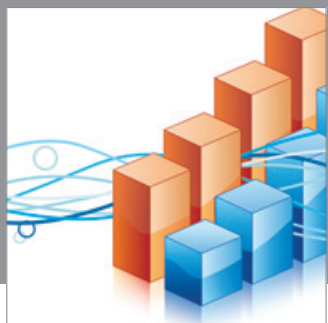

Advances in

Operations Research

mansans

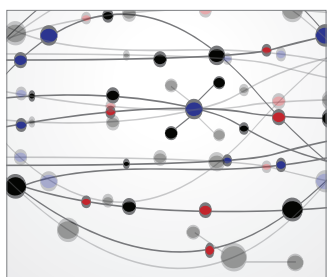

The Scientific World Journal
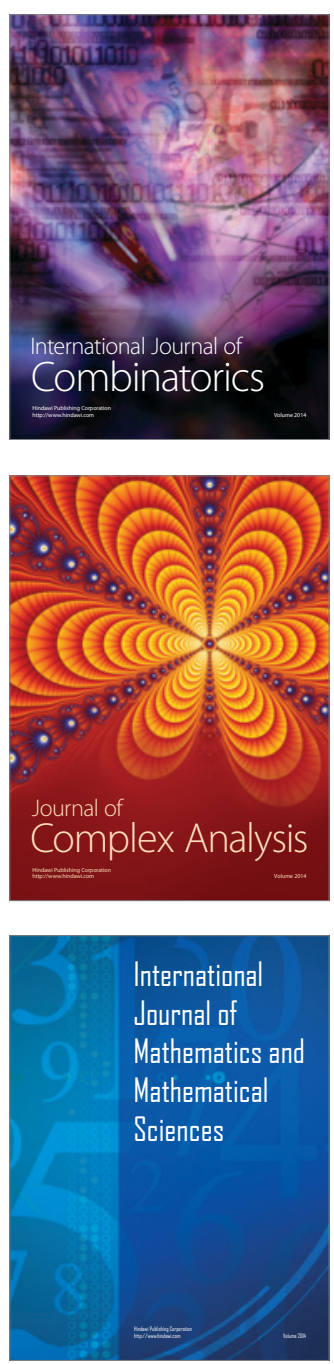
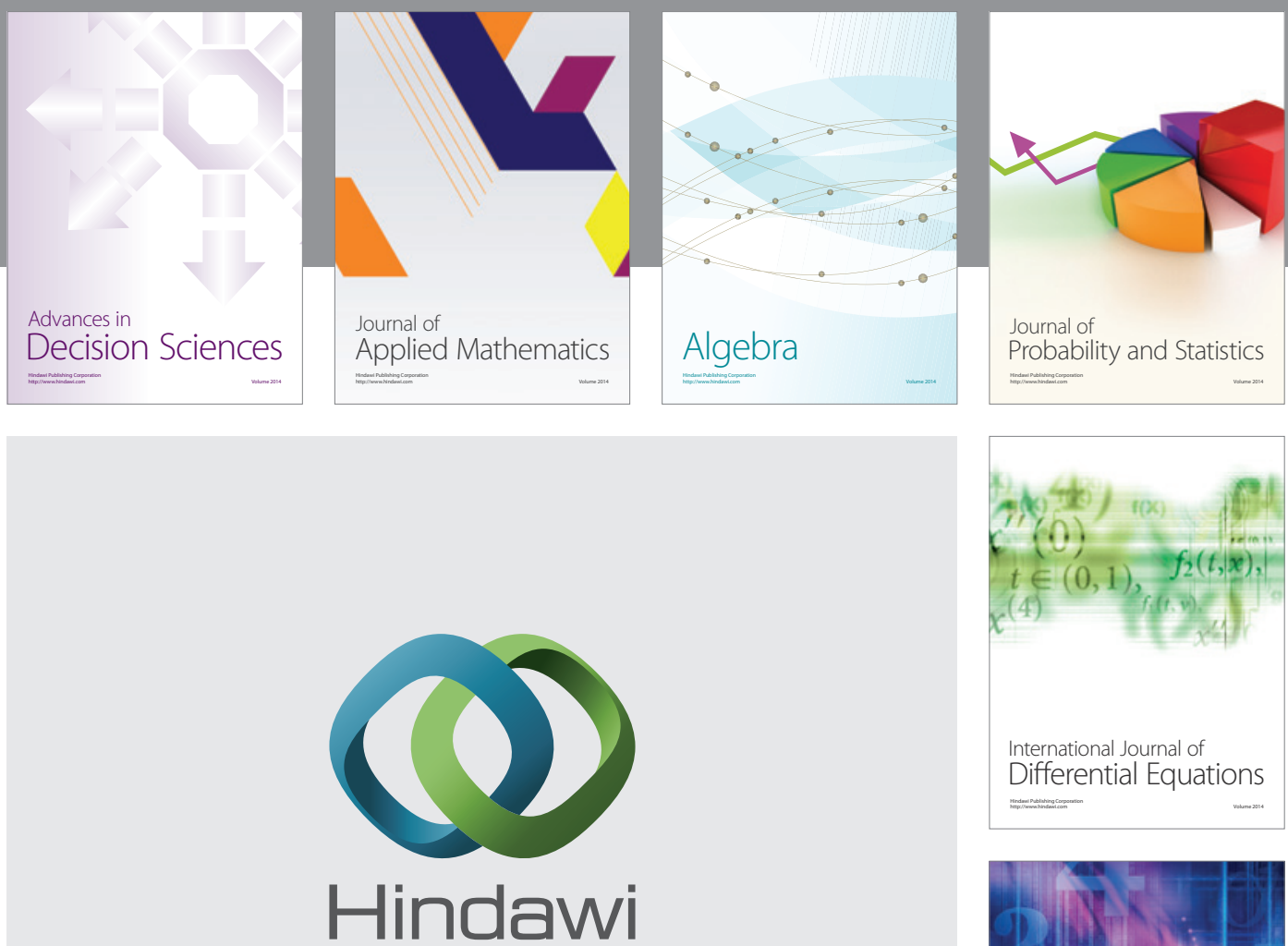

Submit your manuscripts at http://www.hindawi.com
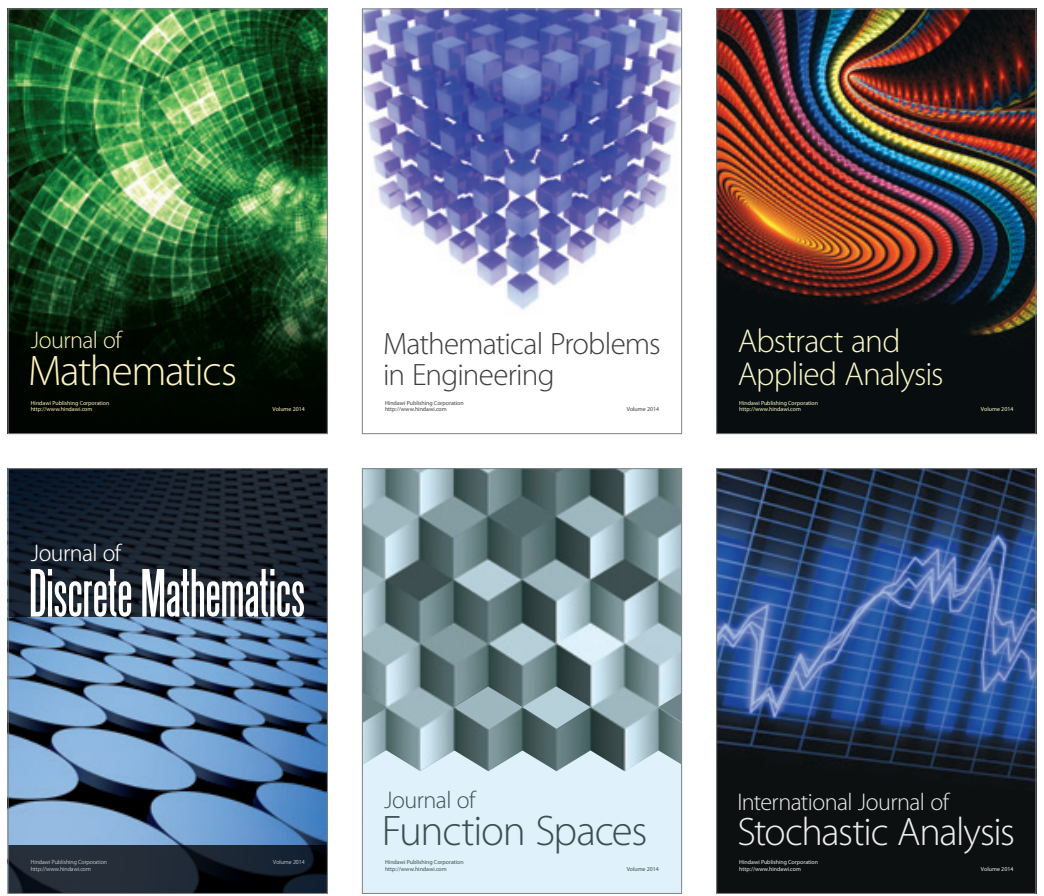

Journal of

Function Spaces

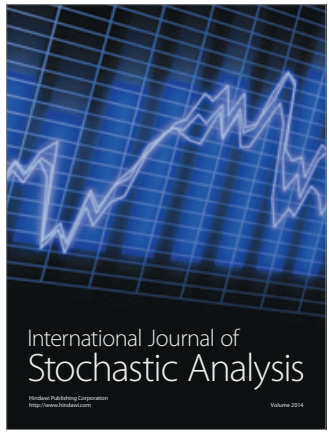

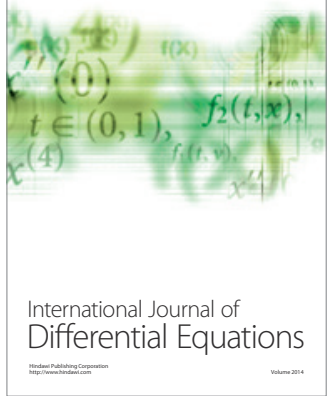
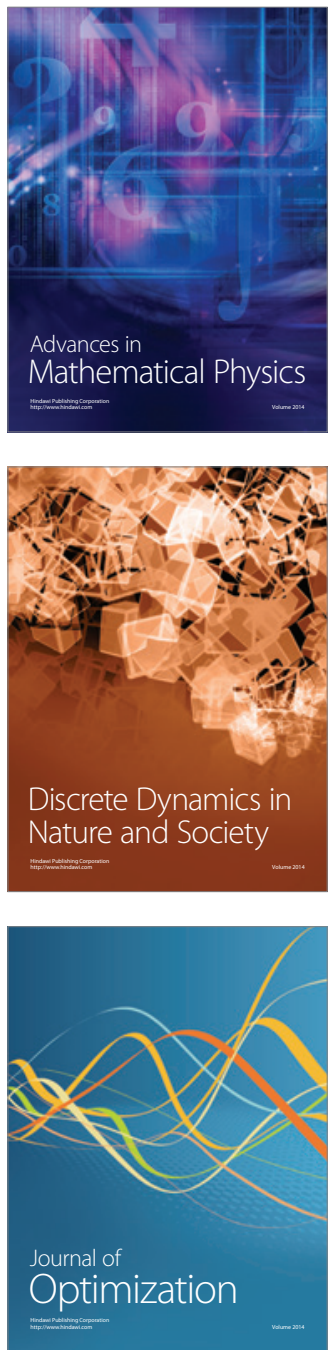